\title{
Significance of Cryo-Scanning Electron Microscopy (Cryo-SEM) in Evaluating the Morphology of Multivesicular Liposomes
}

Soumyarwit Manna ${ }^{1,2}$, Yong $\mathrm{Wu}^{2}$, Bonhye $\mathrm{Koo}^{1,2}$, Xiaoming $\mathrm{Xu}^{3}$, Stephanie Choi ${ }^{1}$, Yan Wang ${ }^{1}$, Darby Kozak $^{1}$ and Jiwen Zheng ${ }^{2 *}$

${ }^{1 .}$ Division of Therapeutic Performance, Office of Research Standards, Office of Generic Drugs, Center for Drug Evaluation and Research, Food and Drug Administration, Silver Spring, MD, USA.

2. Division of Biology, Chemistry and Materials Science, Office of Science and Engineering

Laboratories, Center for Devices and Radiological Health, Food and Drug Administration, Silver Spring, MD, USA.

${ }^{3 .}$ Division of Product Quality Research, Office of Testing and Research, Office of Pharmaceutical Quality, Center for Drug Evaluation and Research, Food and Drug Administration, Silver Spring, MD, USA.

* Corresponding author: Jiwen.zheng@fda.hhs.gov

Multivesicular liposome (MVL) is a lipid-based drug delivery platform that provides sustained release of drugs with short half-lives. It can be differentiated structurally and morphologically from traditional lamellar liposomes based on their characteristic inner polyhedral honeycomb structures. This characteristic polyhedral structure of the MVL platform facilitates improved drug encapsulation, prolonged drug release duration and enhanced structural stability. Currently, there are three MVL-based complex drug products (also commonly known as the DepoFoam technology) approved by the FDA. To demonstrate pharmaceutical equivalence and bioequivalence to the reference listed drug (RLD), it is imperative for the generic products to show comparative physicochemical properties (Q3 sameness), in addition to the qualitative/quantitative (Q1/Q2) sameness. It has been reported that the fusion, collapse or rearrangement of the characteristic polyhedral lipid bilayer structures can potentially cause a change in the release mechanism of the active pharmaceutical ingredient (API) from the MVL formulation. Therefore, it is critical to develop a robust method to evaluate the native morphology and size distribution of the MVLs, which could also reveal the morphological changes over the course of the API release from the MVLs.

Currently, the methods employed to determine the morphology and size distribution of the MVL particles include Laser Diffraction (LD), Optical Microscopy (OM), Confocal Microscopy (CM) and Electron Microscopy (EM). LD and OM are limited to measuring the outer dimensions of the MVLs and fail to measure the dimensions of the characteristic inner polyhedral honeycomb structures of the MVLs. In contrast, CM (Fig. 1A) and EM offer improved direct imaging of the characteristic inner polyhedral honeycomb structures. Particularly, cryo-scanning electron microscopy (cryo-SEM) is one of the suitable techniques as it preserves the hydrated structure of the MVLs and provides an accurate highresolution representation of the MVL structures (Figs. 1B-1D).

In this study, we have compared the morphology of MVLs when observed with a) cryo-SEM and b) traditional room temperature SEM. As observed in cryo-SEM, the mean diameter of the spherical MVLs and characteristic inner polyhedral honeycomb structures were $23.6 \pm 11.5 \mu \mathrm{m}$ and $1.52 \pm 0.44 \mu \mathrm{m}$, respectively (Figs.1B-1D). The outer dimensions of the spherical MVLs, as observed in the cryo-SEM were consistent to measurements gathered from LD and CM. In comparison, traditional room temperature SEM showed the mean diameter of the spherical MVLs to be $3.0 \pm 1.1 \mu \mathrm{m}$ (Figs. 1E-1F) 
and $3.2 \pm 1.9 \mu \mathrm{m}$ (Figs. $1 \mathrm{G}-1 \mathrm{H})$, when the MVL formulation was dried at room temperature $\left(\sim 25^{\circ} \mathrm{C}\right)$ and at $37^{\circ} \mathrm{C}$, respectively. These measurements are almost an order of magnitude lesser than that estimated from CM, LD and cryo-SEM. Furthermore, traditional room temperature SEM did not reveal any inner polyhedral honeycomb structures of the MVLs, which was probably caused by dehydration of the liposomal structures during sample preparation.

Our findings reveal the importance of cryo-SEM technique in appropriate estimation of the morphology of the MVLs in comparison to traditional room temperature SEM method. It also offers advantage of high-resolution imaging of both the outer structure and the characteristic inner polyhedral honeycomb structure, in comparison to LD and CM. Such an accurate methodology may be used as prospective technique to establish equivalence of physicochemical properties between RLD and generic products [4].

\section{References:}

[1] S Manna et al., J. Control. Release 294 (2019), p 279.

[2] P Chahar and K Cumminngs III, J. Pain Res 5 (2012), p. 257.

[3] S Mantripragada, Prog Lipid Res 41(5) (2002), p. 392.

[4] This project was supported in part by an appointment to the Research Participation Program at Center for Drug Evaluation and Research administered by the Oak Ridge Institute for Science and Education through an agreement between the U.S. Department of Energy and FDA. The authors would like to acknowledge FDA Advanced Characterization Facility (ACF), CDRH/OSEL/DBCMS and CDER/OPQ/OTR for instrument use. Soumyarwit Manna, Yong Wu and Bonhye Koo were supported in part by an appointment to the Research Participation Program at the U.S. Food and Drug Administration (FDA), administered by the Oak Ridge Institute for Science and Education through an interagency agreement between the U.S. Department of Energy and the FDA.

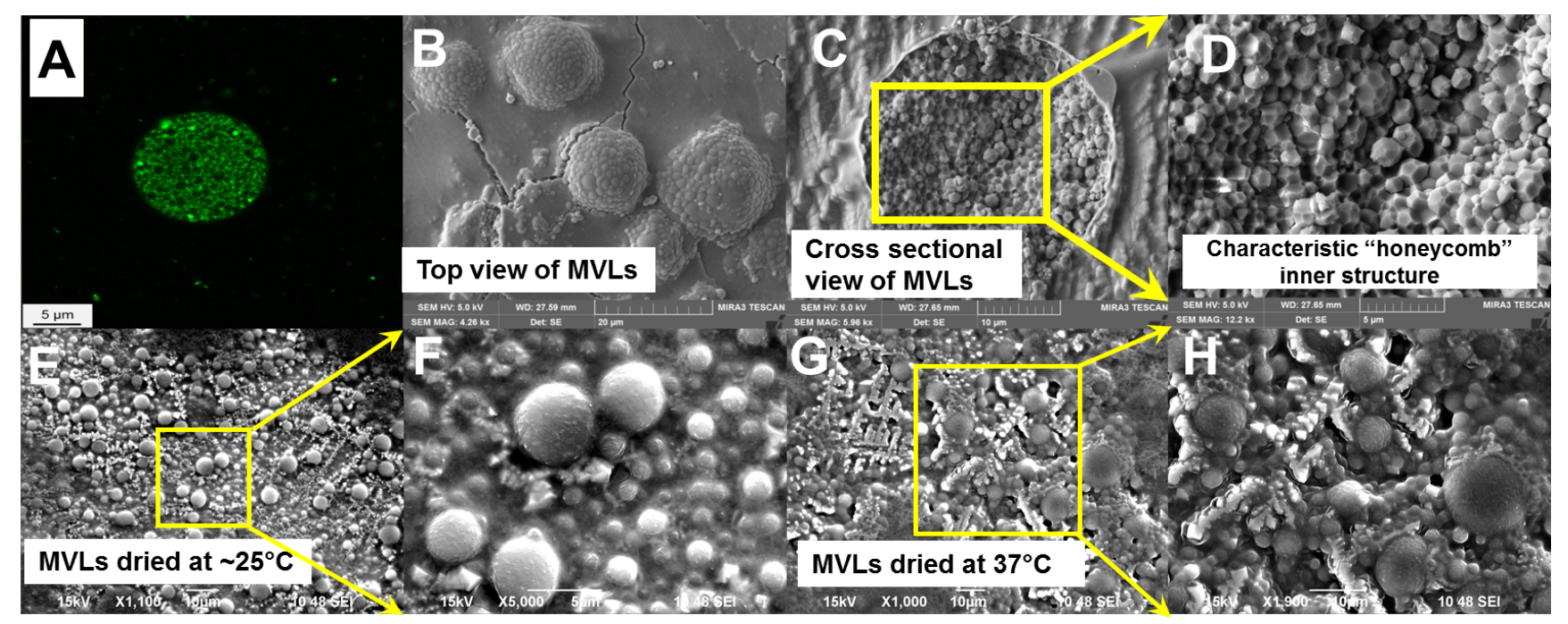

Figure 1. Confocal microscopy (A) and cryo-SEM (B) showing the top view of MVLs. Cryo-SEM showing the $\mathrm{C}$ ) cross-sectional view and D) inner structure of MVLs. Room temperature SEM imaging showing the MVLs dried at $\sim 25^{\circ} \mathrm{C}(\mathrm{E}-\mathrm{F})$ and dried at $37^{\circ} \mathrm{C}(\mathrm{G}-\mathrm{H})$. 